\title{
Cerebellar and hindbrain motion in Chiari malformation with and without syringomyelia
}

\author{
Vannessa Leung, BSc (Med), BMed MD, ${ }^{1}$ John S. Magnussen, MBBS, PhD, FRANZCR, ${ }^{2}$ \\ Marcus A. Stoodley, MBBS, PhD, FRACS, ${ }^{2}$ and Lynne E. Bilston, BE, MSE, PhD ${ }^{3}$ \\ ${ }^{1}$ Faculty of Medicine, University of New South Wales; ${ }^{2}$ Faculty of Medicine and Health Sciences, Macquarie University; and \\ ${ }^{3}$ Neuroscience Research Australia and Prince of Wales Clinical School, University of New South Wales, Sydney, Australia
}

\begin{abstract}
OBJECTIVE The pathogenesis of syringomyelia associated with Chiari malformation type I (CM-I) is unclear. Theories of pathogenesis suggest the cerebellar tonsils may obstruct CSF flow or alter pressure gradients, or their motion might act as a piston to increase CSF pressure in the spinal subarachnoid space. This study was performed to measure cerebellar tonsillar and hindbrain motion in CM-I and assess the potential contributions to syrinx formation.
\end{abstract}

METHODS Sixty-four CM-I patients and 25 controls were retrospectively selected from a clinical database, and all subjects had undergone cardiac-gated cine balanced fast-field echo MRI. There were a total of 36 preoperative CM-I scans, which consisted of 15 patients with and 21 patients without syringomyelia. Nineteen patients underwent paired pre- and postoperative imaging. Anteroposterior (AP) and superoinferior (SI) movements of the tip of the cerebellar tonsils, obex, fastigium of the fourth ventricle, pontomedullary junction, and cervicomedullary junction were measured. The distance between the fastigium and tip of the tonsils was used to calculate tonsillar tissue strain $\left(\Delta i / i_{0}\right)$.

RESULTS CM-I patients had significantly greater cerebellar tonsillar motion in both the AP and SI directions than controls (AP $+0.34 \mathrm{~mm}[+136 \%], p<0.001 ; \mathrm{SI}+0.49 \mathrm{~mm}[+163 \%], p<0.001$ ). This motion decreased after posterior fossa decompression (AP $-0.20 \mathrm{~mm}[-33 \%], p=0.001 ; \mathrm{SI}-0.29 \mathrm{~mm}[-36 \%] ; p<0.001$ ), but remained elevated above control levels $(\mathrm{AP}+56 \%, p=0.021 ; \mathrm{SI}+67 \%, p=0.015)$. Similar trends were seen for all other tracked landmarks. There were no significant differences in the magnitude or timing of motion throughout the hindbrain between $\mathrm{CM}-\mathrm{I}$ patients with and without syringomyelia. Increased tonsillar tissue strain correlated with Valsalva headaches $(p=0.03)$.

CONCLUSIONS Cerebellar tonsillar motion may be a potential marker of CM-I and may have use in tailoring surgical procedures. The lack of association with syringomyelia suggests that tonsillar motion alone is not the driver of syrinx formation. Tonsillar tissue strain may play a part in the pathophysiology of Valsalva headaches.

http://thejns.org/doi/abs/10.3171/2015.8.SPINE15325

KEY WORDS Chiari malformation; syringomyelia; cerebellar tonsils; balanced fast-field echo; brain motion; cervical

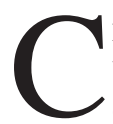
HIARI malformation type I (CM-I) is a craniocervical junction abnormality marked by elongation and ectopia of the cerebellar tonsils into the spinal canal. ${ }^{8} \mathrm{CM}$-I patients commonly develop syringomyelia, although the true prevalence is not well defined (rates in the literature vary between $23 \%$ and $80 \%)$. $^{1,17-19,33,34,47,50,52,54}$ Surgical treatment with posterior fossa decompression (PFD) is not universally successful, with failure of symptomatic resolution reported in up to a third of cases. . $^{17,42,43,61}$ The pathogenesis of syringomyelia in CM-I (and other etiologies) remains unclear, which makes it difficult to develop improved treatments.
A plethora of theories have been postulated to explain the pathogenesis of syringomyelia. In particular, the Oldfield et al..$^{40}$ piston theory and the Greitz ${ }^{22}$ intramedullary pulse pressure theory share the idea that cerebellar tonsillar motion is the primary driving factor behind syrinx formation. The Oldfield theory speculates that exaggerated tonsillar "thrust" imparts heightened CSF pressure waves into the spinal subarachnoid space (SSAS), which forces CSF to flow along the perivascular spaces into the spinal cord. ${ }^{40}$ The Greitz theory speculates that narrowing of the foramen magnum with tonsillar motion induces a Venturi effect that enlarges the cord radially and encourages ex-

ABBREVIATIONS AP = anteroposterior; $\mathrm{bFFE}=$ balanced fast-field echo; $\mathrm{CLM}=$ Chiari-like malformation; $\mathrm{CM}-\mathrm{I}=$ Chiari malformation Type I; GRASS = gradient recalled acquisition in steady state; $\mathrm{PC}=$ phase contrast; $\mathrm{PFD}=$ posterior fossa decompression; $\mathrm{SI}$ = superoinferior; $\mathrm{SSAS}=$ spinal subarachnoid space.

SUBMITTED March 15, 2015. ACCEPTED August 11, 2015.

INCLUDE WHEN CITING Published online January 1, 2016; DOI: 10.3171/2015.8.SPINE15325. 
tracellular fluid accumulation..$^{22}$ In light of these theories, measurements of cerebellar tonsillar motion are required to better understand their potential contribution to syringomyelia pathogenesis.

The motion of the cerebellar tonsils (caudal in systole and rostral in diastole) was qualitatively described in earlier studies as being greater in CM-I patients using intraoperative ultrasound, ${ }^{29,40}$ presaturation bolus-tracking MRI ${ }^{53}$ and cine phase-contrast (PC) MRI. ${ }^{59}$ Tonsillar motion existed in both control subjects and CM-I patients, although at a velocity that was 10 times slower in the control group. ${ }^{59}$ Quantitative studies later confirmed significantly increased cerebellar tonsillar pulsation in CM-I patients using gradient recalled acquisition in steady state (GRASS) MRI ${ }^{44}$ and steady-state cine MRI. ${ }^{6,11}$ The recent improvements in cine MRI technology have also enabled the first comparisons between CM-I patients with and without syringomyelia. Cousins and Haughton ${ }^{11}$ reported cerebellar tonsillar motion to be $22 \%$ greater in CM-I patients with syrinx than in those without syrinx (no p values were calculated), whereas Bunck et al..$^{5}$ found no significant differences between groups. The small sample sizes of both studies (11 CM-I and $20 \mathrm{CM}-\mathrm{I}$ patients, respectively) limited the study power, and both assumed that tonsillar motion only occurs in the superoinferior direction. Whether tonsillar motion "normalizes" following PFD, like CSF flow abnormalities in CM-I, 2,4,13,25,28 and accompanies subsequent clinical improvement has not previously been quantified and is of interest. Therefore, cerebellar tonsillar motion needs to be better characterized.

Cine balanced fast-field echo (bFFE) MRI is a relatively new steady-state free precession imaging technique that offers fully refocused transverse magnetization. ${ }^{39}$ Cine bFFE is suitable for neuroimaging, ${ }^{36,45,55}$ and specific applications to that manual measurement of cerebellar tonsillar motion demonstrated substantial to near-perfect reliability ${ }^{48}$ Driver et al. ${ }^{16}$ assessed cerebellar motion using cine bFFE in a related canine condition, Chiari-like malformation (CLM). Pulsation of the entire neuraxis was observed, and cerebellar movement was significantly greater in CLM animals with syringomyelia than those without syrinxes and the control group.

The magnitude and pattern of cerebellar tonsillar and hindbrain motion in humans with CM-I measured using cine bFFE MRI has not yet been reported. The aim of this study was to measure tonsillar and hindbrain motion in CM-I patients with and without syringomyelia using cine bFFE MRI, explore the associations with clinical features/ surgical outcomes, and discern potential contributions to syringomyelia pathogenesis.

\section{Methods}

This study analyzed a previously collected set of imaging data using cross-sectional and paired before-and-after comparisons. Ethical approval was obtained from the Macquarie University Human Research Ethics Committee.

\section{Participants}

The Macquarie University Hospital radiology database was interrogated for craniocervical junction cine bFFE scans. This sequence has been routinely performed at our institution in conjunction with PC and anatomical MR imaging since 2011 on subjects referred for suspected CMI. Independently verified CM-I diagnosis was based on a tonsillar descent $\geq 5 \mathrm{~mm}$ as well as evidence of obstructed CSF spaces. Subjects with basilar invagination were excluded. Sixty-four CM-I patients (mean age 32.7 years; $72 \%$ female) and 25 controls (mean age 32.0 years; $68 \%$ female) were identified. Of the $64 \mathrm{CM}-\mathrm{I}$ patients, $36 \mathrm{had}$ preoperative cine bFFE images: 15 with syringomyelia (mean age 27.9 years; $80 \%$ female) and 21 patients without syringomyelia (mean age 34.2 years; $76 \%$ female). Syringomyelia was defined as an intramedullary cavity with a maximum dimension of at least $50 \%$ of the cord diameter or a cavity with evidence of dissection into the surrounding cord, regardless of the rostrocaudal extent of the cavity. Of these $36 \mathrm{CM}-\mathrm{I}$ patients, 19 underwent PFD (suboccipital craniectomy, duraplasty, and C-1 laminectomy) and had both preoperative and postoperative scans. A separate subgroup of 6 patients presented for secondary ("revision") decompressive surgery following clinical and radiological failure of an initial procedure performed elsewhere. Preand post-revision images were available for the patients who underwent resection of the original graft, division of arachnoid adhesions, and insertion of a pericranial dural graft that was secured to a titanium cranioplasty in an effort to prevent retethering of the graft to the cerebellum. All patients presented with suboccipital headaches (although severity and duration varied), Valsalva headaches, sensory disturbances, and dizziness, although no consistent differences in the signs and symptoms existed between groups. Clinical changes after surgery were not analyzed. Syrinx size was recorded as larger, unchanged, or reduced in size.

\section{Imaging Sequence}

All images were acquired using 3-T MRI (Magnetom; Siemens). Pulse oximetry or electrocardiography triggers were used for retrospective cardiac gating. The cine bFFE sequence was obtained as a single, midsagittal, 4-mm-thick slice with 25 images during the cardiac cycle. Other typical imaging parameters were flip angle $37^{\circ}$, matrix $160 \times 240$, field of view $140 \times 140$, TR 39.3 msec, TE $1.77 \mathrm{msec}$, and in-plane spatial resolution 0.4375 $\mathrm{mm}^{2}$. The typical acquisition time for the complete sequence was approximately 3 minutes ( 2 minutes 30 seconds to 4 minutes 30 seconds) with variations dependent on the subject's heart rate. Cardiac cycles with significant beat-to-beat variability were automatically rejected from the acquisition. Details regarding the individual image frames' relationship to the cardiac cycle were recorded as a percentage of the $\mathrm{R}-\mathrm{R}$ interval.

\section{Data Analysis}

The cine bFFE sequence was reviewed as a loop. Five anatomical landmarks in the sagittal plane were identified: the tip of the tonsils, the obex, the fastigium of the fourth ventricle, the anterior border of the pontomedullary junction, and the anterior border of the cervicomedullary junction. A representative, single-frame, preoperative, CM-I bFFE image is shown in Fig. 1 with the anatomi- 
cal landmarks indicated. Motion tracking was performed manually by a single blinded investigator. Automatic contrast adjustment was applied to permit better visualization of the structures. Manual tracking using image analysis software ImageJ (National Institutes of Health) with plugin MTrackJ (Erik Meijering; Erasmus MC) involved identification of the anatomical landmarks in each consecutive frame. Anteroposterior (AP) and superoinferior (SI) movement were measured for all anatomical landmarks as an average of 3 readings.

The following features were measured from the displacement-time plots: maximal cranial and caudal displacement relative to the initial position; and amount of displacement and timing of these features relative to the cardiac cycle (percent of R-R interval). The portion of the cardiac cycle spent caudally displaced relative to initial tonsillar position was calculated by linear interpolation. The velocity of tonsillar motion was calculated using consecutive magnitudes of displacement (velocity $=\Delta$ distance $/$ $\Delta$ time). Maximum cranial and caudal tonsillar velocities and respective timings relative to the cardiac cycle were noted.

The distance $(l)$ between the fastigium of the fourth ventricle $\left(\mathrm{x}_{1}, \mathrm{y}_{1}\right)$ and tip of the tonsils $\left(\mathrm{x}_{2}, \mathrm{y}_{2}\right)$ was measured over the cardiac cycle in order to characterize tonsillar tissue deformation (Eq. 1).

$$
l=\sqrt{\left(x_{2}-x_{1}\right)^{2}+\left(y_{2}-y_{1}\right)^{2}}
$$

The change in this length $(\Delta l)$ was divided by the initial length $\left(l_{0}\right)$ to calculate tonsillar tissue strain (Strain $=\Delta l / l_{0}$ ).

The presence of suboccipital headaches, headache worsening with Valsalva maneuvers, sensory disturbance, and dizziness were noted at the time of presentation and collated from the clinical files.

The degree of tonsillar herniation was measured perpendicularly from the tip of the tonsils to the basion-opisthion line for each CM-I patient. Imaging alignment was verified by calculating the degree of neck flexion. This was determined by the angulation of the odontoid process, as measured counter-clockwise from the horizontal plane.

\section{Statistical Analysis}

Statistical analysis was performed using SPSS 21.0 (IBM). One-way ANOVA was performed for each motion, timing, velocity, strain, and neck flexion parameter. To test the effect of surgical intervention and CM-I status, control subjects were compared with the pre- and postoperative CM-I groups. To test the effect of syringomyelia, the control subjects were compared with the CM-I with syringomyelia and CM-I without syringomyelia groups. Significant effects in the ANOVA models were investigated with Bonferroni post hoc test of multiple comparisons in order to identify significant differences between individual groups.

Separate paired t-tests were used to compare pre- and postoperative scans from the same patient $(\mathrm{n}=19)$ and pre- and post-revision scans from the same patient $(\mathrm{n}=$ 6 ). Unpaired t-tests were performed to examine the as-

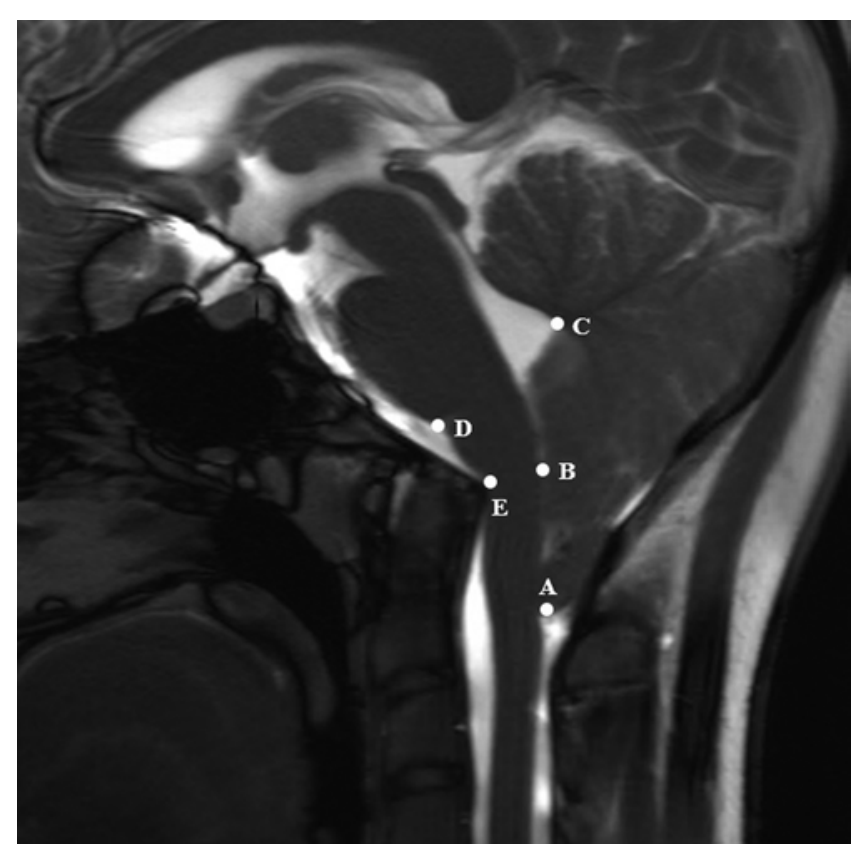

FIG. 1. Representative preoperative bFFE scan of a CM-I patient. The anatomical landmarks of interest are the tip of the cerebellar tonsils (A), the obex $(B)$, the fastigium of the fourth ventricle $(C)$, the anterior margin of the pontomedullary junction (D), and the anterior margin of the cervicomedullary junction (E).

sociation between tonsillar velocity or tissue strain and presenting symptoms. Unpaired t-tests were also used to compare degree of tonsillar herniation between CM-I patients with and without syringomyelia. Data are presented as the mean $\pm \mathrm{SD}$, and $\mathrm{p}<0.05$ was considered statistically significant.

\section{Results}

All syrinx cavities were larger than $50 \%$ of the cord diameter and ranged from 2 spinal levels to extensive holocord cavities. Syrinx size was reduced in all patients who were managed surgically, including the 5 revision surgery patients who presented with recurrent syringomyelia.

There was no significant difference in the magnitude of tonsillar herniation between CM-I patients with and without syringes $(11.5 \pm 5.2 \mathrm{~mm}$ vs $9.6 \pm 6.0 \mathrm{~mm} ; \mathrm{p}=0.322$, unpaired t-test). The angulation of the odontoid process did not differ significantly between the controls and either CM-I group (controls $84.00^{\circ} \pm 5.43^{\circ}, \mathrm{CM}-\mathrm{I}$ with syrinx $84.72^{\circ} \pm 4.73^{\circ}, \mathrm{CM}-\mathrm{I}$ without syrinx $84.94^{\circ} \pm 7.12^{\circ} ; \mathrm{p}=$ 0.909, 1-way ANOVA).

\section{Cerebellar Tonsillar and Hindbrain Motion}

Sagittal cine bFFE MRI sequences permitted clear visualization of cerebellar tonsillar and hindbrain motion in all subjects. A set of typical paired patient preoperative and postoperative cine bFFE sequences are provided as Video 1 and Video 2, respectively.

VIDEO 1. Sample preoperative cine bFFE sequence from a CM-I patient. Copyright Neuroscience Research Australia. Used with permission. Click here to view. 
VIDEO 2. Sample postoperative cine bFFE sequence from the same CM1 patient shown in Video 1. Copyright Neuroscience

Research Australia. Used with permission. Click here to view.

The manual tracking method produced high reliability with a Cronbach's alpha value of 0.831 , which is comparable to results of the validation study by Sharma et al. ${ }^{48}$

\section{Effect of CM-I on Motion}

All tracked anatomical points moved periodically during the cardiac cycle in both the AP and SI directions. Motion was greater in CM-I patients than controls for all landmarks in both the AP and SI directions (Table 1). Figure 2 shows the movement of the tip of the cerebellar tonsils was $0.34 \mathrm{~mm}(136 \%)$ greater in the AP direction $(\mathrm{p}<0.001)$ and $0.49 \mathrm{~mm}(163 \%)$ greater in the SI direction $(\mathrm{p}<0.001)$ for preoperative CM-I patients in comparison with controls.

\section{Effect of PFD Surgery on Motion}

The cross-sectional results, illustrated in Fig. 2, show PFD reduced cerebellar tonsillar motion by $0.20 \mathrm{~mm}$ $(33 \%)$ in the AP direction $(\mathrm{p}=0.001)$ and $0.29 \mathrm{~mm}(36 \%)$ in the SI direction $(\mathrm{p}<0.001)$ in comparison with preoperative CM-I patients. Postoperative motion remained elevated above the control levels in both the AP $(p=0.021)$ and SI $(\mathrm{p}=0.015)$ directions. A similar trend was observed pre- and postoperatively for the other 4 anatomical points, although the postoperative and control values were not significantly different (Tables 1 and 2).

In the subgroup of patients with paired pre- and postoperative scans $(\mathrm{n}=19)$, a significant reduction in cerebellar tonsillar motion was observed in the AP $(0.76 \pm 0.28 \mathrm{~mm}$ vs $0.42 \pm 0.19 \mathrm{~mm} ; \mathrm{p}<0.001)$ and SI $(0.99 \pm 0.36 \mathrm{~mm}$ vs $0.51 \pm 0.21 \mathrm{~mm} ; \mathrm{p}<0.001)$ directions. A similar relationship existed for the other 4 anatomical landmarks in both directions (AP and SI) as illustrated in Fig. 3.

In the subgroup of patients undergoing revision PFD, no significant difference in cerebellar tonsillar motion was apparent before and after the second procedure in the AP $(0.47 \pm 0.36 \mathrm{~mm}$ vs $0.43 \pm 0.30 \mathrm{~mm} ; \mathrm{p}=0.432)$ or SI $(0.68$ $\pm 0.49 \mathrm{~mm}$ vs $0.53 \pm 0.35 \mathrm{~mm} ; \mathrm{p}=0.153$ ) directions. $\mathrm{A}$ similar relationship existed for the 4 other landmarks in both directions (AP and SI).

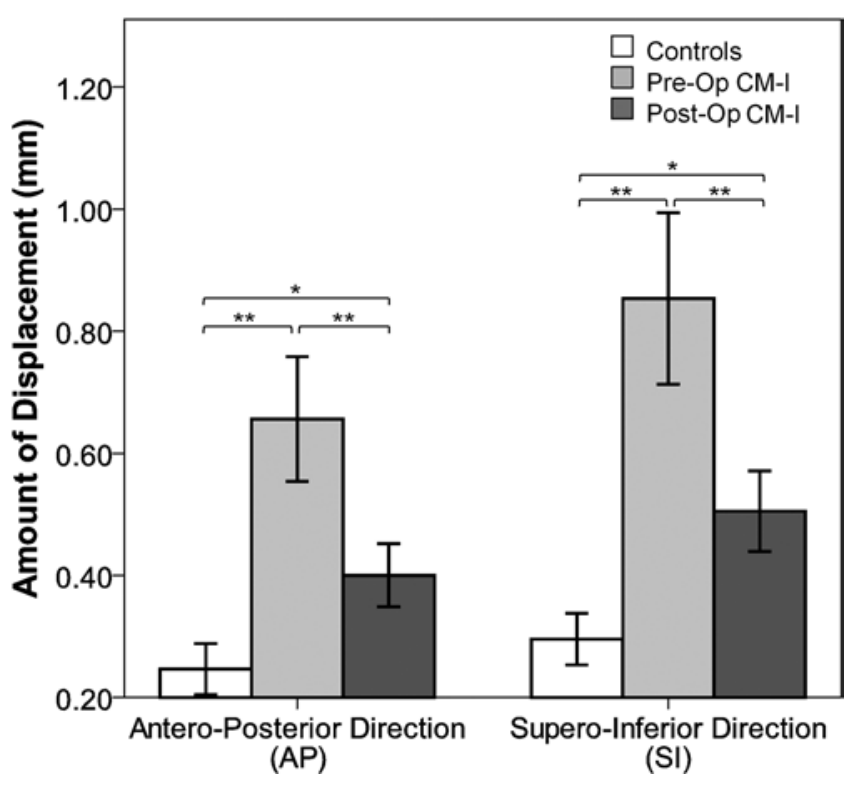

FIG. 2. Displacement of the tip of the cerebellar tonsils for all groups. Group mean values with error bars indicating the $95 \% \mathrm{Cls}$ according to 1-way ANOVA with Bonferroni post hoc testing for the control (white), preoperative CM-I patient (light gray), and postoperative patient (dark gray) groups. Bar clusters represent motion in the AP and SI directions. ${ }^{*} p<0.05 ;{ }^{* *} p<0.001$.

\section{Motion in Syringomyelia Patients}

The presence of a syrinx had no effect on the displacement of the 5 tracked anatomical landmarks in both the $\mathrm{AP}$ and SI directions. Cerebellar tonsillar motion in the SI direction averaged $0.81 \pm 0.47 \mathrm{~mm}$ for CM-I patients with syringomyelia and $0.88 \pm 0.40 \mathrm{~mm}$ for CM-I patients without syringomyelia $(\mathrm{p}=0.57)$.

\section{Timing of Tonsillar Motion}

CM-I, syringomyelia, and operative status had no effect on the timing of maximum cranial or caudal tonsillar displacement or relative time spent caudally displaced.

Velocity of Tonsillar Motion

CM-I patients without syringomyelia had faster maxi-

TABLE 1. Summary of the maximum displacement of tracked anatomical landmarks for all groups

\begin{tabular}{lccc}
\hline & & Mean \pm SD $(\mathrm{mm})$ \\
\cline { 2 - 4 } \multicolumn{1}{c}{ Parameter } & Control & Preop CM-I & Postop CM-I \\
\hline Tonsils (AP) & $0.25 \pm 0.10$ & $0.59 \pm 0.30$ & $0.39 \pm 0.22$ \\
\hline Tonsils (SI) & $0.30 \pm 0.10$ & $0.79 \pm 0.45$ & $0.50 \pm 0.28$ \\
\hline Obex (AP) & $0.26 \pm 0.10$ & $0.40 \pm 0.19$ & $0.33 \pm 0.15$ \\
\hline Obex (SI) & $0.36 \pm 0.14$ & $0.70 \pm 0.33$ & $0.46 \pm 0.18$ \\
\hline 4th ventricle fastigium (AP) & $0.24 \pm 0.10$ & $0.42 \pm 0.28$ & $0.27 \pm 0.11$ \\
\hline 4th ventricle fastigium (SI) & $0.29 \pm 0.10$ & $0.53 \pm 0.26$ & $0.36 \pm 0.12$ \\
\hline Pontomedullary junction (AP) & $0.32 \pm 0.12$ & $0.56 \pm 0.29$ & $0.35 \pm 0.11$ \\
\hline Pontomedullary junction (SI) & $0.33 \pm 0.11$ & $0.58 \pm 0.31$ & $0.42 \pm 0.16$ \\
\hline Cervicomedullary junction (AP) & $0.30 \pm 0.11$ & $0.44 \pm 0.22$ & $0.31 \pm 0.13$ \\
\hline Cervicomedullary junction (SI) & $0.32 \pm 0.17$ & $0.60 \pm 0.32$ & $0.36 \pm 0.18$ \\
\hline
\end{tabular}

Tonsils $=$ tip of the cerebellar tonsils. 
TABLE 2. Summary of ANOVA and Bonferroni multiple comparisons tests of the cross-sectional results for maximum displacement of the tracked anatomical landmarks

\begin{tabular}{lccccc}
\hline & \multicolumn{4}{c}{$p$ Value } \\
\cline { 2 - 3 } \multicolumn{1}{c}{ Parameter } & ANOVA & & \multicolumn{2}{c}{ Bonferroni Multiple Comparisons } \\
\cline { 2 - 3 } \cline { 5 - 6 } & $\begin{array}{c}\text { CM-l/Operative } \\
\text { Status Overall }\end{array}$ & & $\begin{array}{c}\text { Control vs Preop } \\
\text { CM-I }\end{array}$ & $\begin{array}{c}\text { Preop CM-I vs } \\
\text { Postop CM-I }\end{array}$ & $\begin{array}{c}\text { Control vs Postop } \\
\text { CM-I }\end{array}$ \\
\hline Tonsils (AP) & $<0.001$ & & $<0.001$ & 0.001 & 0.021 \\
\hline Tonsils (SI) & $<0.001$ & & $<0.001$ & $<0.001$ & 0.015 \\
\hline Obex (AP) & $<0.001$ & & 0.002 & 0.001 & 0.066 \\
\hline Obex (SI) & $<0.001$ & & $<0.001$ & $<0.001$ & 0.201 \\
\hline 4th ventricle fastigium (AP) & $<0.001$ & & 0.001 & 0.001 & 0.767 \\
\hline 4th ventricle fastigium (SI) & $<0.001$ & & $<0.001$ & $<0.001$ & 0.309 \\
\hline Pontomedullary junction (AP) & $<0.001$ & & $<0.001$ & $<0.001$ & 0.736 \\
\hline Pontomedullary junction (SI) & $<0.001$ & & $<0.001$ & 0.003 & 0.117 \\
\hline Cervicomedullary junction (AP) & 0.001 & & 0.004 & 0.003 & 0.99 \\
\hline Cervicomedullary junction (SI) & $<0.001$ & & $<0.001$ & $<0.001$ & 0.844 \\
\hline
\end{tabular}

mum caudally directed tonsillar velocities than controls $(+6.07 \pm 3.25 \mathrm{~cm} / \mathrm{sec} v \mathrm{vs}+3.20 \pm 2.07 \mathrm{~cm} / \mathrm{sec} ; \mathrm{p}=0.004$ 1-way ANOVA with Bonferroni post hoc test). The maximum caudal tonsillar velocity in the CM-I patients with syringomyelia was not different from that in CM-I patients without syringomyelia or controls. The maximum rostral tonsillar velocity was not significantly different between groups. Velocities in the postoperative group were not significantly different to preoperative or control groups. No significant differences in the timing of maximal cranial or caudal tonsillar velocity were detected between groups.

Maximum caudally directed tonsillar velocity was significantly greater in the subgroup of CM-I patients reporting Valsalva headaches than those without $(+6.8 \pm 3.34 \mathrm{~m} /$ sec vs $+4.52 \pm 1.98 \mathrm{~m} / \mathrm{sec} ; \mathrm{p}=0.027)$. No other associations between clinical presentation and tonsillar velocity or timing were noted.

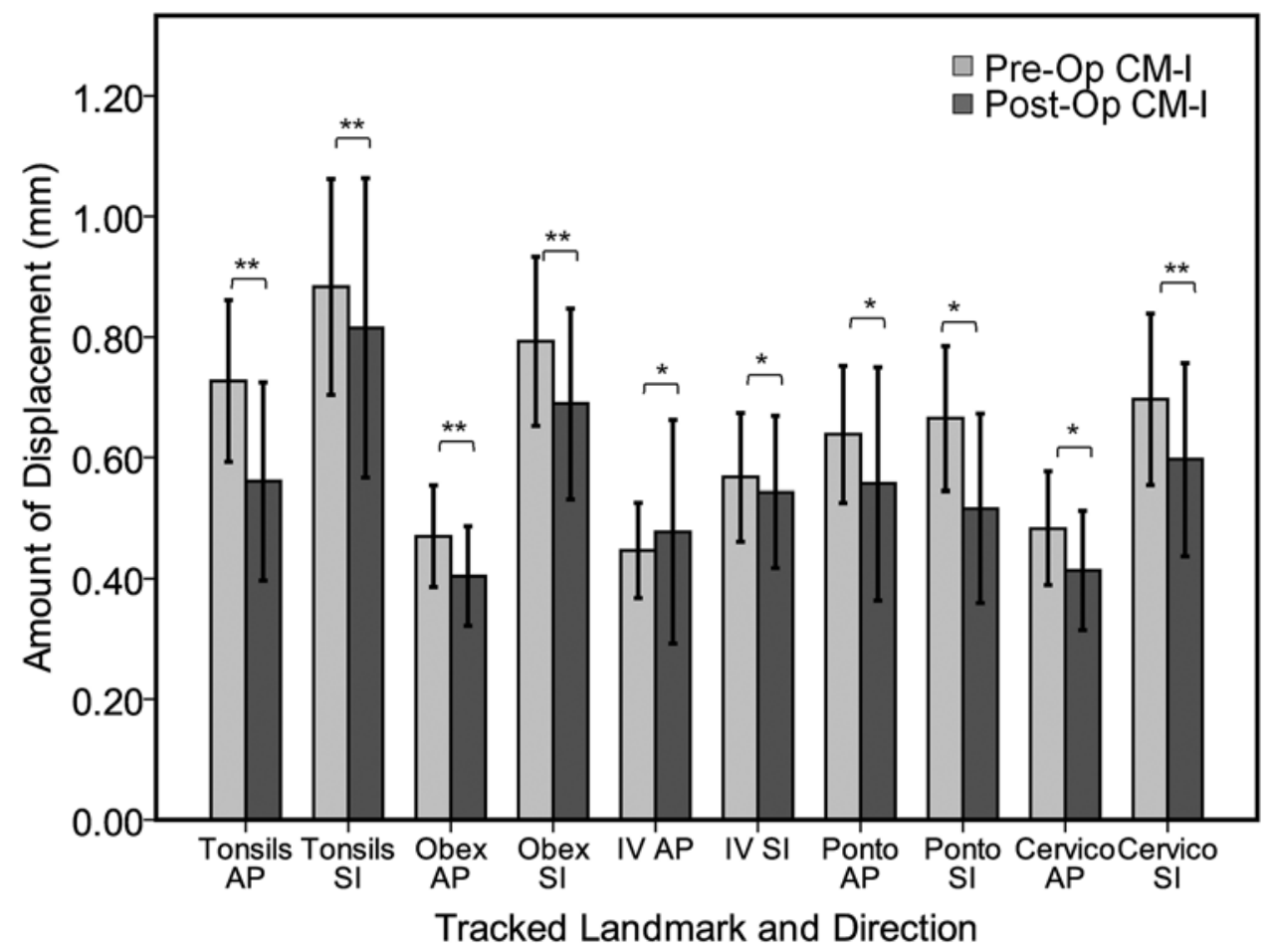

FIG. 3. Paired pre- and postoperative comparison of the displacement of all tracked anatomical structures. Group mean values with the error bars indicating $95 \%$ Cls are shown. The asterisks indicate ${ }^{*} p<0.05$ and ${ }^{* *} p<0.001$ from paired t-tests of the preoperative (light gray) and postoperative (dark gray) groups. Bar clusters represent each anatomical landmark in both the AP and SI directions. Abbreviations: Cervico = cervicomedullary junction; IV = fastigium of the fourth ventricle; Ponto = pontomedullary junction; Tonsils $=$ tip of the cerebellar tonsils. 


\section{Tonsillar Tissue Strain}

Figure 4 shows that the tissue strain on the cerebellar tonsils was significantly greater in preoperative CM-I patients than in controls $(\mathrm{p}=0.001)$. Tissue strain was reduced significantly postoperatively $(p=0.003)$ to levels similar to those in the control group. Tonsillar tissue strain was not significantly different between CM-I patients with and without syringomyelia.

Tonsillar tissue strain was significantly higher in the subgroup of CM-I patients reporting Valsalva headaches than those without $(0.015 \pm 0.007$ vs $0.009 \pm 0.006 ; p=$ $0.03)$. The presence of suboccipital headaches, sensory disturbance, and dizziness was not associated with tissue strain.

\section{Discussion}

This study provides the first quantitative evidence that cerebellar tonsillar and hindbrain motion reduces and "normalizes" after PFD. The extent of tonsillar pulsation was not significantly different between CM-I patients with and without syringomyelia.

\section{Cerebellar Tonsillar Motion in CM-I Patients and Controls}

The results of this study confirm greater cerebellar tonsillar and hindbrain motion in CM-I patients (regardless of syringomyelia status) in comparison with controls. This motion occurred in both the AP and SI directions and was most pronounced for SI displacement of the tip of the tonsils. Tonsillar and hindbrain motion may be explained by the Monro-Kellie doctrine of constant total intracranial volume. ${ }^{23}$ In control subjects, normal systolic

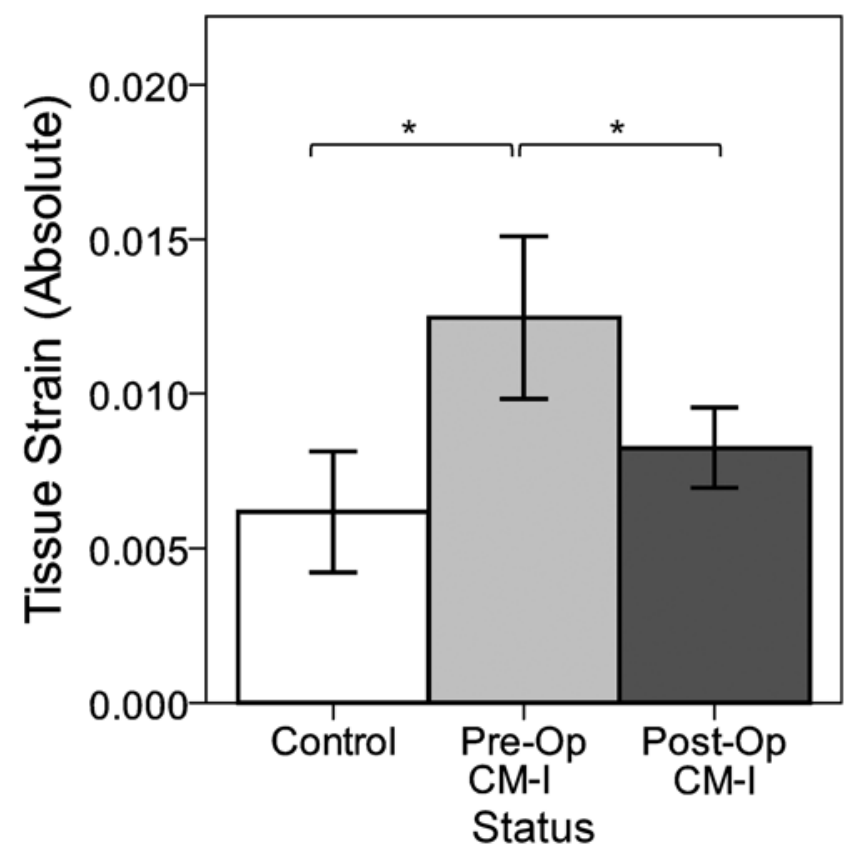

FIG. 4. Cerebellar tissue strains as measured from the fastigium of the fourth ventricle to the tip of the tonsils for all groups. Group mean values with error bars indicating $95 \% \mathrm{Cls}$ are shown, and the asterisks indicate ${ }^{*} p<0.05$ according to 1-way ANOVA with Bonferroni post hoc testing for the control (white), preoperative CM-I patient (light gray), and postoperative patient (dark gray) groups. brain expansion (as cardiac output reaches the calvaria) is accommodated by caudal CSF flow. In CM-I patients, a small posterior cranial fossa results in tonsillar herniation and the obstruction of CSF flow at the foramen magnum; therefore, brain expansion must be (partially) accommodated by other means such as inferior displacement of the cerebellar tonsillar tissue. ${ }^{25,40}$ Our findings are in agreement with those of other intraoperative ultrasound ${ }^{29,40}$ and MRI studies in humans..$^{11,44,48,53,59}$ However, in the somewhat analogous canine CLM, only a subgroup of the CLM animals (those with syringomyelia) demonstrated significantly increased motion over control animals. ${ }^{16}$ Whether differences between the human and canine conditions or differences between dog breeds (control group) explain these differences is unknown. Since tonsillar ectopia length (the current CM-I diagnostic marker) correlates poorly with clinical symptoms, ${ }^{57}$ further studies should investigate if cerebellar tonsillar motion may have use as an adjunct marker of CM-I.

\section{Effect of Surgery on Tonsillar Motion}

Our results suggest that cerebellar tonsillar and hindbrain motion is reduced following PFD. This was confirmed by a significant reduction in postoperative motion (across all landmarks) seen with the paired analysis of patients with both pre- and postoperative scans. Interestingly, of all landmarks, despite a reduction, only postoperative tonsillar motion (and not those of the other 4 anatomical landmarks) remained elevated above control levels. To the best of our knowledge, this is the first quantification of cerebellar tonsillar motion postsurgery. These observations complement qualitative intraoperative ultrasound studies describing reductions in tonsillar pulsation following dural opening. ${ }^{25,40} \mathrm{CM}-\mathrm{I}$ is associated with a small posterior fossa volume, ${ }^{3,38,51,54}$ and, since PFD normalizes both cerebellar tonsillar shape ${ }^{25,42}$ and motion (current study), this suggests that both occur secondary to an underdeveloped posterior fossa. Interestingly, in our subset of patients undergoing revision PFD (after clinical and radiological failure of the original PFD surgery), no significant difference in tonsillar motion was noted after the second (clinically successful) procedure. This may be the result of adhesions that arise from the initial surgery limiting tonsillar movement. While Heiss et al. ${ }^{26}$ reported reduced tonsillar pulsation in this category of patients using intraoperative ultrasound at the second surgery, the possible influence of anesthesia and different extent of adhesions is unknown. Cerebellar tonsillar motion reduces after surgery and may be a tool for pre- and intraoperative tailoring of surgical intervention. Prospective studies should assess its predictive capacity for surgical success and clinical outcomes.

\section{Association Between Cerebellar Tonsillar Motion and Syringomyelia}

This study identified no significant quantitative differences in the degree or timing of cerebellar tonsillar pulsation between CM-I patients with and without a syrinx. Tonsillar motion reflects displaced volume from the cranial cavity during the cardiac cycle and is a balance of 2 parameters: cranial volume and cardiac output (to the cranium). Morphometric and anatomical studies indicate no 
differences in cranial volume between CM-I patients with and without syringes, as demonstrated by similar degrees of crowding (reflected by the posterior fossa/intracranial volume ratio), ${ }^{54}$ cross-sectional CSF area at the foramen magnum, ${ }^{5}$ and degree of tonsillar ectopia (as demonstrated in the current study). ${ }^{5,34}$ No anecdotal or experimental data suggest that cardiac output differs between CM-I patients with and without syringomyelia. Therefore, assuming similar cardiac input to the cranium, no difference in (cerebellar tonsillar) volume displaced is expected. These findings are consistent with those in other studies in humans that report no significant differences in tonsillar pulsation between CM-I patients with and without syringomyelia using GRASS PC, ${ }^{44}$ fast imaging employing steady-state acquisition (FIESTA), ${ }^{11}$ and cine bFFE MRI. ${ }^{5}$

In contrast, a canine bFFE study of the somewhat analogous condition, CLM, documented increased cerebellar motion in CLM dogs with syringomyelia in comparison with CLM dogs without syringomyelia. ${ }^{16}$ Whether anesthesia or other differences between humans and dogs influenced these findings is unclear. Different degrees of head flexion are known to alter cerebellar herniation length in CLM, ${ }^{56}$ and it is unknown if this is true for CM-I or cerebellar tonsillar motion in humans. Different degrees of head flexion between (but not within) our subjects in comparison with Driver et al.'s canine groups may also account for the dissimilar results. Interestingly, the average tonsillar motion (CLM/CM-I with syrinx values given) was much greater and more variable in dogs $(4.18 \pm$ $2.63 \mathrm{~mm}$ according to Driver et al. $\left.{ }^{16}\right)$ than that in humans (current study $0.81 \pm 0.47 \mathrm{~mm}$; Bunck et al. $.^{5} 1.0 \pm 0.6 \mathrm{~mm}$; Cousins and Haughton ${ }^{11} 0.61 \pm 0.03 \mathrm{~mm}$ ) despite similar or smaller degrees of herniation. ${ }^{12,14,31}$ Whether increased cerebellar motion has a different effect on the canine system is unknown. Morphometric studies further suggest a probable difference between human and canine conditions. CLM canines with syringomyelia display shallower caudal cranial fossa measurements, ${ }^{7}$ overcrowding of the caudal cranial fossa by an increased cerebellar volume, ${ }^{49}$ and greater volume of brain parenchyma situated in the caudal cranial fossa ${ }^{15}$ than CLM canines without syringomyelia. Limited morphometric studies in humans have not made such associations with syringomyelia..$^{42,54,60}$ It is therefore unknown how applicable and analogous canine CLM is to CM-I in humans and if the contributions of cerebellar pulsations to syringomyelia pathogenesis are similar.

The findings of this study suggest that cerebellar tonsillar pulsation is greater in CM-I patients, but not associated with syringomyelia. These results call into question whether tonsillar motion alone plays a major role in syringomyelia pathogenesis. Oldfield et al. ${ }^{40}$ and Greitz $^{22}$ both proposed theories of syringomyelia pathogenesis, in which cerebellar tonsillar motion is one of the key driving factors. The Oldfield "piston" theory speculates that exaggerated tonsillar "thrust" imparts large CSF pressure waves into the SSAS, which pushes CSF into the cord via perivascular spaces. ${ }^{40}$ The Greitz intramedullary pulse pressure theory speculates that exaggerated tonsillar motion narrows the foramen magnum and induces the Venturi effect, which distends the spinal cord and encourages extracellular fluid accumulation. ${ }^{22}$ The finding that there is no dif- ference in the degree or timing of tonsillar motion between CM-I patients with and without syringomyelia suggests that this alone is not the differentiating factor. The results, therefore, suggest that it is unlikely that cerebellar tonsillar motion plays a key role in syrinx pathogenesis in the absence of systematic differences in SSAS compliance. For the proposed theories to be confirmed, similar degrees of tonsillar motion must impart greater CSF pressures in patients who develop syringomyelia. Mechanically, this would require lower SSAS compliance in CM-I patients who develop syringes than in those who do not. Measures of CSF pressures and SSAS compliance would be useful for further testing of these theories. Furthermore, it is possible that cerebellar tonsillar motion may have differed between syringomyelia and nonsyringomyelia CM-I patients early in syrinx formation, but this could not be evaluated within the design of the current study. No anecdotal or experimental observations suggest that tonsillar motion changes over time, although this has been speculated in canine CLM. ${ }^{16}$ Longitudinal studies that follow CM-I patients as they develop syringomyelia may provide insight into whether any changes to cerebellar motion occur with time and whether this contributes to syringomyelia pathogenesis.

\section{Association Between Cerebellar Tonsillar Velocity and Syringomyelia}

The results presented here indicate that CM-I patients without syringomyelia have significantly faster peak caudal tonsillar velocity than controls. CM-I patients with syringomyelia, however, did not differ significantly from either group. The differences in maximum caudal tonsillar velocity may reflect SSAS resistance to tonsillar displacement. In CM-I patients without syringomyelia, there is relatively normal anatomy caudal to the foramen magnum. With each cardiac systole, sudden volume displacement in the calvaria induces rapid "thrust" of the tonsils and thus a heightened maximum tonsillar velocity. In CM-I patients with syringomyelia, the presence of a syrinx increases the cross-sectional area of the spinal cord ${ }^{27}$ and reduces the size of the SSAS, thereby increasing resistance in the SSAS. During cardiac systole, tonsillar motion meets this resistance and achieves a lower maximum velocity. Interestingly, the phenomenon in which CM-I patients without syringomyelia differ more from controls than CM-I patients with syringomyelia has also been reported in terms of various CSF flow parameters., ${ }^{9,10}$ Further studies are needed to assess if these tonsillar velocity findings are (causally) associated with CSF findings and if any implications exist for syringomyelia pathogenesis.

\section{Tonsillar Tissue Strain and Strain Rate}

This study also found greater cerebellar tonsillar tissue deformation (strain) in CM-I patients, which reduces to control levels following PFD. Tonsillar motion, therefore, involves deformation and stretching of tonsillar tissue during each cardiac cycle rather than just simple SI displacement. Mechanical deformation of the tonsils or associated structures may be a mechanism underlying some of the clinical features of CM-I, although this has not been previously examined. In support of this, we found an as- 
sociation between Valsalva-induced headaches, strain, and tonsillar velocity. Several theories explaining the pathophysiology of Valsalva headaches have been proposed, including transient craniospinal pressure dissociation that irritates the arachnoid and tonsillar blood vessels, ${ }^{58} \mathrm{com}-$ pression of the $\mathrm{C} 1$ and $\mathrm{C} 2$ nerve roots by further herniation of the cerebellar tonsils during Valsalva maneuvers, ${ }^{41}$ reduced CSF compliance due to a small posterior cranial fossa volume, ${ }^{34}$ and raised intrathecal pressures that stretch the spinal dura ${ }^{46}$ While our study was not designed to explore these aspects, our results suggest a possible role of mechanical strain in Valsalva headache pathophysiology. Biomechanical studies have demonstrated that tissue damage is both strain magnitude and strain rate dependent..$^{20,35}$ Imaging studies have reported exaggerated brainstem motion during Valsalva maneuvers. ${ }^{32}$ Our results suggest that CM-I patients who develop Valsalva headaches have higher resting tonsillar tissue strains and caudal tonsillar velocities than CM-I patients without Valsalva headaches. During the Valsalva maneuvers, the added strain acting on the tonsillar tissue or associated vessels and nerves may be sufficient to exceed a threshold, leading to headache in the former but not the latter group. Prospective studies should further assess tonsillar tissue strain and deformation in different directions and investigate if other adjacent structures (e.g., vessels, nerves) are subject to the same stresses.

\section{Limitations}

In interpreting the results of this study, it is important to consider its limitations and assumptions. First, while the current study has demonstrated increased cerebellar tonsillar motion in CM-I patients in comparison with controls, the control group was not comprised of age- and sex-matched healthy volunteers. However, there were no significant differences in the baseline characteristics between the groups (age, sex, degree of head flexion), and the control subjects were assessed to be without any structural or CSF abnormalities by an independent radiologist. Second, the cross-sectional nature of this study did not allow us to characterize if cerebellar tonsillar motion changed over the course of the condition. Although no differences in tonsillar motion were detected between CM-I patients with and without syringomyelia at the time of these scans, it is possible a difference may have existed earlier during syrinx formation. We were also unable to follow up patients to observe if syringomyelia developed at a later date. However, our results were consistent with other studies and, to date, no evidence shows that tonsillar motion changes over time. Third, the association between tonsillar motion and severity and duration of clinical outcomes could not be assessed in this study. CM-I with and without syringomyelia has a variable clinical presentation, ${ }^{18,21,34,47}$ and no valid tool exists to measure this. A validated and reliable Chiari-specific outcome tool, such as that being developed by Mueller and Oro, ${ }^{37}$ may allow these associations to be investigated in future studies. This may also provide insight into the future utility of cerebellar tonsillar motion for predicting patient response to surgery, or for tailoring surgical procedures. Fourth, the cine bFFE images used in this study were peripherally triggered, and thus variable lengths of trigger delay may have affected the accuracy of the timing parameters despite reporting them as portion of the R-R cardiac interval. Electrocardiographic gating in future prospective studies may be considered. Finally, several theories of syringomyelia pathogenesis postulate that cerebellar tonsillar motion alters CSF flow and pressure patterns,,$^{22,40}$ and thus concurrent measurement of tonsillar motion and CSF flow is warranted. The retrospective nature of this current study meant that the CSF flow images were of insufficient quality and consistency for flow measurements to be confidently made despite our original intent. While differences in CSF flow velocity ${ }^{5,10,24,30}$ and timing $2,5,10,17,30$ have been well documented, their relationship with tonsillar motion is of interest and should be investigated with future prospective studies.

\section{Conclusions}

This study characterized cerebellar tonsillar and hindbrain motion in CM-I patients with and without syringomyelia. CM-I patients had exaggerated tonsillar and hindbrain motion, which were reduced postoperatively to near control levels. There was no difference in the timing or magnitude of motion between CM-I patients with and without syringomyelia. These findings suggest that cerebellar tonsillar motion alone is not the driver of syrinx formation, adding more understanding to current theories of pathogenesis. Increased tonsillar tissue strain and strain rates may underlie the pathophysiology of Valsalva headaches in CM-I. This study has also demonstrated the robustness and utility of cine bFFE techniques when applied routinely in the clinical setting.

\section{Acknowledgments}

This work was supported in part by the Column of Hope Foundation. Lynne Bilston is supported by an NHMRC senior research fellowship.

\section{References}

1. Amer TA, el-Shmam OM: Chiari malformation type I: a new MRI classification. Magn Reson Imaging 15:397-403, 1997

2. Armonda RA, Citrin CM, Foley KT, Ellenbogen RG: Quantitative cine-mode magnetic resonance imaging of Chiari I malformations: an analysis of cerebrospinal fluid dynamics. Neurosurgery 35:214-224, 1994

3. Badie B, Mendoza D, Batzdorf U: Posterior fossa volume and response to suboccipital decompression in patients with Chiari I malformation. Neurosurgery 37:214-218, 1995

4. Brugières P, Idy-Peretti I, Iffenecker C, Parker F, Jolivet O, Hurth M, et al: CSF flow measurement in syringomyelia. AJNR Am J Neuroradiol 21:1785-1792, 2000

5. Bunck AC, Kroeger JR, Juettner A, Brentrup A, Fiedler B, Crelier GR, et al: Magnetic resonance 4D flow analysis of cerebrospinal fluid dynamics in Chiari I malformation with and without syringomyelia. Eur Radiol 22:1860-1870, 2012

6. Bunck AC, Kröger JR, Jüttner A, Brentrup A, Fiedler B, Schaarschmidt F, et al: Magnetic resonance 4D flow characteristics of cerebrospinal fluid at the craniocervical junction and the cervical spinal canal. Eur Radiol 21:1788-1796, 2011

7. Carrera I, Dennis R, Mellor DJ, Penderis J, Sullivan M: Use of magnetic resonance imaging for morphometric analysis of the caudal cranial fossa in Cavalier King Charles Spaniels. Am J Vet Res 70:340-345, 2009

8. Chiari H: Concerning alterations in the cerebellum result- 
ing from cerebral hydrocephalus. 1891. Pediatr Neurosci 13:3-8, 1987

9. Clarke EC, Fletcher DF, Stoodley MA, Bilston LE: Computational fluid dynamics modelling of CSF pressure in Chiari malformation and syringomyelia. J Biomech 46:1801-1809, 2013

10. Clarke EC, Stoodley MA, Bilston LE: Changes in temporal flow characteristics of CSF in Chiari malformation Type I with and without syringomyelia: implications for theory of syrinx development. J Neurosurg 118:1135-1140, 2013

11. Cousins J, Haughton V: Motion of the cerebellar tonsils in the foramen magnum during the cardiac cycle. AJNR Am J Neuroradiol 30:1587-1588, 2009

12. Couturier J, Rault D, Cauzinille L: Chiari-like malformation and syringomyelia in normal cavalier King Charles spaniels: a multiple diagnostic imaging approach. J Small Anim Pract 49:438-443, 2008

13. Dolar MT, Haughton VM, Iskandar BJ, Quigley M: Effect of craniocervical decompression on peak CSF velocities in symptomatic patients with Chiari I malformation. AJNR Am J Neuroradiol 25:142-145, 2004

14. Driver CJ, De Risio L, Hamilton S, Rusbridge C, Dennis R, McGonnell IM, et al: Changes over time in craniocerebral morphology and syringomyelia in cavalier King Charles spaniels with Chiari-like malformation. BMC Vet Res 8:215, 2012

15. Driver CJ, Rusbridge C, Cross HR, McGonnell I, Volk HA: Relationship of brain parenchyma within the caudal cranial fossa and ventricle size to syringomyelia in cavalier King Charles spaniels. J Small Anim Pract 51:382-386, 2010

16. Driver CJ, Watts V, Bunck AC, Van Ham LM, Volk HA: Assessment of cerebellar pulsation in dogs with and without Chiari-like malformation and syringomyelia using cardiac-gated cine magnetic resonance imaging. Vet J 198:88-91, 2013

17. Ellenbogen RG, Armonda RA, Shaw DWW, Winn HR: Toward a rational treatment of Chiari I malformation and syringomyelia. Neurosurg Focus 8(3):E6, 2000

18. Elster AD, Chen MYM: Chiari I malformations: clinical and radiologic reappraisal. Radiology 183:347-353, 1992

19. Furtado SV, Thakre DJ, Venkatesh PK, Reddy K, Hegde AS: Morphometric analysis of foramen magnum dimensions and intracranial volume in pediatric Chiari I malformation. Acta Neurochir (Wien) 152:221-227, 2010

20. Geddes DM, Cargill RS II, LaPlaca MC: Mechanical stretch to neurons results in a strain rate and magnitude-dependent increase in plasma membrane permeability. J Neurotrauma 20:1039-1049, 2003

21. George TM, Higginbotham NH: Defining the signs and symptoms of Chiari malformation type I with and without syringomyelia. Neurol Res 33:240-246, 2011

22. Greitz D: Unraveling the riddle of syringomyelia. Neurosurg Rev 29:251-264, 2006

23. Greitz D, Wirestam R, Franck A, Nordell B, Thomsen C, Ståhlberg F: Pulsatile brain movement and associated hydrodynamics studied by magnetic resonance phase imaging. The Monro-Kellie doctrine revisited. Neuroradiology 34:370 380,1992

24. Haughton VM, Korosec FR, Medow JE, Dolar MT, Iskandar BJ: Peak systolic and diastolic CSF velocity in the foramen magnum in adult patients with Chiari I malformations and in normal control participants. AJNR Am J Neuroradiol 24:169-176, 2003

25. Heiss JD, Patronas N, DeVroom HL, Shawker T, Ennis R, Kammerer W, et al: Elucidating the pathophysiology of syringomyelia. J Neurosurg 91:553-562, 1999

26. Heiss JD, Suffredini G, Smith R, DeVroom HL, Patronas NJ, Butman JA, et al: Pathophysiology of persistent syringomyelia after decompressive craniocervical surgery. Clinical article. J Neurosurg Spine 13:729-742, 2010
27. Hofmann E, Warmuth-Metz M, Bendszus M, Solymosi L: Phase-contrast MR imaging of the cervical CSF and spinal cord: volumetric motion analysis in patients with Chiari I malformation. AJNR Am J Neuroradiol 21:151-158, 2000

28. Iskandar BJ, Quigley M, Haughton VM: Foramen magnum cerebrospinal fluid flow characteristics in children with Chiari I malformation before and after craniocervical decompression. J Neurosurg 101 (2 Suppl):169-178, 2004

29. Jokich PM, Rubin JM, Dohrmann GJ: Intraoperative ultrasonic evaluation of spinal cord motion. J Neurosurg 60:707711,1984

30. Liu B, Wang ZY, Xie JC, Han HB, Pei XL: Cerebrospinal fluid dynamics in Chiari malformation associated with syringomyelia. Chin Med J (Engl) 120:219-223, 2007

31. Lu D, Lamb CR, Pfeiffer DU, Targett MP: Neurological signs and results of magnetic resonance imaging in 40 cavalier King Charles spaniels with Chiari type 1-like malformations. Vet Rec 153:260-263, 2003

32. Maier SE, Hardy CJ, Jolesz FA: Brain and cerebrospinal fluid motion: real-time quantification with M-mode MR imaging. Radiology 193:477-483, 1994

33. Mauer UM, Gottschalk A, Mueller C, Weselek L, Kunz U, Schulz C: Standard and cardiac-gated phase-contrast magnetic resonance imaging in the clinical course of patients with Chiari malformation Type I. Neurosurg Focus 31(3):E5, 2011

34. Milhorat TH, Chou MW, Trinidad EM, Kula RW, Mandell $\mathrm{M}$, Wolpert C, et al: Chiari I malformation redefined: clinical and radiographic findings for 364 symptomatic patients. Neurosurgery 44:1005-1017, 1999

35. Miller K, Chinzei K: Mechanical properties of brain tissue in tension. J Biomech 35:483-490, 2002

36. Moon WJ, Roh HG, Chung EC: Detailed MR imaging anatomy of the cisternal segments of the glossopharyngeal, vagus, and spinal accessory nerves in the posterior fossa: the use of 3D balanced fast-field echo MR imaging. AJNR Am J Neuroradiol 30:1116-1120, 2009

37. Mueller DM, Oro' JJ: The Chiari Symptom Profile: development and validation of a Chiari-/syringomyelia-specific questionnaire. J Neurosci Nurs 45:205-210, 2013

38. Nishikawa M, Sakamoto H, Hakuba A, Nakanishi N, Inoue Y: Pathogenesis of Chiari malformation: a morphometric study of the posterior cranial fossa. J Neurosurg 86:40-47, 1997

39. Nitz WR: Fast and ultrafast non-echo-planar MR imaging techniques. Eur Radiol 12:2866-2882, 2002

40. Oldfield EH, Muraszko K, Shawker TH, Patronas NJ: Pathophysiology of syringomyelia associated with Chiari I malformation of the cerebellar tonsils. Implications for diagnosis and treatment. J Neurosurg 80:3-15, 1994

41. Pascual J, Oterino A, Berciano J: Headache in type I Chiari malformation. Neurology 42:1519-1521, 1992

42. Pillay PK, Awad IA, Little JR, Hahn JF: Symptomatic Chiari malformation in adults: a new classification based on magnetic resonance imaging with clinical and prognostic significance. Neurosurgery 28:639-645, 1991

43. Pinna G, Alessandrini F, Alfieri A, Rossi M, Bricolo A: Cerebrospinal fluid flow dynamics study in Chiari I malformation: implications for syrinx formation. Neurosurg Focus 8(3):E3, 2000

44. Pujol J, Roig C, Capdevila A, Pou A, Martí-Vilalta JL, Kulisevsky J, et al: Motion of the cerebellar tonsils in Chiari type I malformation studied by cine phase-contrast MRI. Neurology 45:1746-1753, 1995

45. Reubelt D, Small LC, Hoffmann MH, Kapapa T, Schmitz BL: MR imaging and quantification of the movement of the lamina terminalis depending on the CSF dynamics. AJNR Am J Neuroradiol 30:199-202, 2009

46. Sansur CA, Heiss JD, DeVroom HL, Eskioglu E, Ennis R, 
Oldfield EH: Pathophysiology of headache associated with cough in patients with Chiari I malformation. J Neurosurg 98:453-458, 2003

47. Sekula RF Jr, Arnone GD, Crocker C, Aziz KM, Alperin N: The pathogenesis of Chiari I malformation and syringomyelia. Neurol Res 33:232-239, 2011

48. Sharma A, Parsons MS, Pilgram TK: Balanced steady-state free-precession MR imaging for measuring pulsatile motion of cerebellar tonsils during the cardiac cycle: a reliability study. Neuroradiology 54:133-138, 2012

49. Shaw TA, McGonnell IM, Driver CJ, Rusbridge C, Volk HA: Increase in cerebellar volume in Cavalier King Charles Spaniels with Chiari-like malformation and its role in the development of syringomyelia. PLoS One 7:e33660, 2012

50. Stevens JM, Serva WA, Kendall BE, Valentine AR, Ponsford JR: Chiari malformation in adults: relation of morphological aspects to clinical features and operative outcome. J Neurol Neurosurg Psychiatry 56:1072-1077, 1993

51. Stovner LJ, Bergan U, Nilsen G, Sjaastad O: Posterior cranial fossa dimensions in the Chiari I malformation: relation to pathogenesis and clinical presentation. Neuroradiology 35:113-118, 1993

52. Strahle J, Muraszko KM, Kapurch J, Bapuraj JR, Garton HJL, Maher CO: Chiari malformation Type I and syrinx in children undergoing magnetic resonance imaging. J Neurosurg Pediatr 8:205-213, 2011

53. Terae S, Miyasaka K, Abe S, Abe H, Tashiro K: Increased pulsatile movement of the hindbrain in syringomyelia associated with the Chiari malformation: cine-MRI with presaturation bolus tracking. Neuroradiology 36:125-129, 1994

54. Trigylidas T, Baronia B, Vassilyadi M, Ventureyra ECG: Posterior fossa dimension and volume estimates in pediatric patients with Chiari I malformations. Childs Nerv Syst 24:329-336, 2008

55. Tsuchiya K, Aoki C, Hachiya J: Evaluation of MR cisternography of the cerebellopontine angle using a balanced fast-field-echo sequence: preliminary findings. Eur Radiol 14:239-242, 2004

56. Upchurch JJ, McGonnell IM, Driver CJ, Butler L, Volk HA Influence of head positioning on the assessment of Chiarilike malformation in Cavalier King Charles spaniels. Vet Rec 169:277, 2011
57. Ventureyra ECG, Aziz HA, Vassilyadi M: The role of cine flow MRI in children with Chiari I malformation. Childs Nerv Syst 19:109-113, 2003

58. Williams B: Cough headache due to craniospinal pressure dissociation. Arch Neurol 37:226-230, 1980

59. Wolpert SM, Bhadelia RA, Bogdan AR, Cohen AR: Chiari I malformations: assessment with phase-contrast velocity MR. AJNR Am J Neuroradiol 15:1299-1308, 1994

60. Yamazaki Y, Tachibana S, Takano M, Fujii K: Clinical and neuroimaging features of Chiari type I malformations with and without associated syringomyelia. Neurol Med Chir (Tokyo) 38:541-547, 1998

61. Zhang Y, Zhang N, Qiu H, Zhou J, Li P, Ren M, et al: An efficacy analysis of posterior fossa decompression techniques in the treatment of Chiari malformation with associated syringomyelia. J Clin Neurosci 18:1346-1349, 2011

\section{Disclosures}

The authors report no conflict of interest concerning the materials or methods used in this study or the findings specified in this paper.

\section{Author Contributions}

Conception and design: Bilston, Stoodley. Acquisition of data: Leung, Magnussen. Analysis and interpretation of data: all authors. Drafting the article: Bilston, Leung. Critically revising the article: all authors. Reviewed submitted version of manuscript: all authors. Approved the final version of the manuscript on behalf of all authors: Bilston. Statistical analysis: Bilston, Leung. Study supervision: Bilston.

\section{Supplemental Information \\ Videos}

Video 1. https://vimeo.com/141546502.

Video 2. https://vimeo.com/141546500.

\section{Correspondence}

Lynne E. Bilston, NeuRA, P.O. Box 1165, Randwick, NSW 2031, Australia.email: 1.bilston@neura.edu.au. 